Pacific Journal of Mathematics

STOCHASTIC INTEGRALS IN ABSTRACT WIENER SPACE 


\section{STOCHASTIC INTEGRALS IN ABSTRACT WIENER SPACE}

\section{HuI-HSIUNG KuO}

Let $W(t, \omega)$ be the Wiener process on an abstract Wiener space $(i, H, B)$ corresponding to the canonical normal distributions on $H$. Stochastic integrals $\int_{0}^{t} \xi(s, \omega) d W(s, \omega)$ and $\int_{0}^{t}(\zeta(s, \omega), d W(s, \omega))$ are defined for non-anticipating transformations $\xi$ with values in $\mathscr{B}(B, B)$ such that $(\xi(t, \omega)-I)(B) \subset$ $B^{*}$ and $\zeta$ with values in $H$. Suppose $X(t, \omega)=x_{0}+$ $\int_{0}^{t} \xi(s, \omega) d W(s, \omega)+\int_{0}^{t} \sigma(s, \omega) d s$, where $\sigma$ is a non-anticipating transformation with values in $H$. Let $f(t, x)$ be a continuous function on $R \times B$, continuously twice differentiable in the $H$-directions with $D^{2} f(t, x) \in \mathscr{B}_{1}(H, H)$ for the $x$ variable and once differentiable for the $t$ variable. Then $f(t, X(t, \omega))=$ $f\left(0, x_{0}\right)+\int_{0}^{t}\left(\xi^{*}(s, \omega) D f(s, X(s, \omega), d W(s, \omega))+\int_{0}^{t}\{\partial f / \partial s(s, X(s, \omega))+\right.$ $<D f\left(s, X(s, \omega), \sigma(s, \omega)>+\frac{1}{2} \operatorname{trace}\left[\xi^{*}(s, \omega) D^{2} f(s, X(s, \omega)) \xi(s, \omega)\right]\right\} d s$, where $<,>$ is the inner product of $H$. Under certain assumptions on $A$ and $\sigma$ it is shown that the stochastic integral equation $X(t, \omega)=x_{0}+\int_{0}^{t} A(X(s, \omega)) d W(s, \omega)+\int_{0}^{t} \sigma(X(s, \omega)) d s$ has a unique solution. This solution is a homogeneous strong Markov process.

1. Introduction. The notion of stochastic integral introduced by $\mathrm{K}$. Ito $[5 ; 8$ ] is well-known nowadays [10]. Its generalization to infinite dimensional space has been investigated and used for the study of differential equations of diffusion type. See, for instance, [1] and [2]. The purpose of this paper is to define and study stochastic integrals with respect to standard Wiener process in an abstract Wiener space [3; 4]. We will generalize Ito's formula [7] and study the stochastic integral equation. We remark that our work is quite different from that of [1] and closely related to that of [2]. However, we have a more general space and weaker assumptions than [2].

2. Abstract Wiener space. In this section we give a brief review of the notion of an abstract Wiener space and establish notation at the same time. Let $H$ be a real separable Hilbert space with norm and inner product denoted by $|\cdot|$ and $\langle$, $\rangle$ respectively. Let $\mu_{t}(t>0)$ be the cylinder set measure on $H$ defined by $\mu_{t}(C)=$ $(2 \pi t)^{-n / 2} \int_{D} \exp \left\{-|x|^{2} / 2 t\right\} d x$, where $C=P^{-1}(D), D$ is a Borel set in the image of an $n$-dimensional projection $P$ in $H$ and $d x$ is Lebesgue measure in $P H$. A measurable norm on $H$ is a norm $\|\cdot\|$ on $H$ with 
the property that for every $\varepsilon>0$ there exists a finite-dimensional projection $P_{c}$ such that $\mu_{1}(\{x \in H ;\|P x\|>\varepsilon\})<\varepsilon$ whenever $P$ is a finite-dimensional projection orthogonal to $P_{0}$. Denote by $B$ the completion of $H$ with respect to $\|\cdot\|$. Let $i$ be the inclusion map from $H$ into $B$ and $j$ the embedding (by restriction) of $B^{*}$ into $H^{*}$. Henceforth we will make the identifications $H^{*} \equiv H$ and $B^{*} \equiv j B^{*}$. Thus $B^{*} \subset H \subset B$ and $\langle x, y\rangle=(x, y)$ for all $x$ in $B^{*}$ and $y$ in $H$, where (, ) denotes the natural pairing of $B^{*}$ and $B$. The triple $(i, H, B)$ is called an abstract Wiener space. It is shown in [3] that $\mu_{t} \circ i^{-1}$, defined on the cylinder sets of $B$, has a unique countably additive extension $p_{t}$ to the Borel field of $B$.

Define for $x$ in $B$ and $E$ a Borel subset of $B, p_{t}(x, E)=p_{t}(E-x)$. It is remarked in [4] that $p_{t}(x, \cdot), t>0$, are the transition probabilities for a Markov process with continuous sample paths starting at the origin in $B$. Throughout this paper $\Omega$ will denote the space of continuous functions $\omega$ from $[0, \infty)$ into $B$ vanishing at 0 . Then there is a unique probability measure $\mathscr{P}$ on the $\sigma$-field $\mathscr{C l}$ generated by the coordinate functions such that if $0=t_{0}<t_{1}<\cdots<t_{n}$ then $\omega\left(t_{j+1}\right)-\omega\left(t_{j}\right), 0 \leqq j \leqq n-1$, are independent and the $j$ th one has distribution measure $p_{t_{j+1}-t_{j}}$. We denote by $\mathscr{E}$ the expectation with respect to $(\Omega, \mathscr{P})$. The process $W(t)$ given by $W(t)(\omega)=\omega(t)$ is called a Wiener process with state space $B$.

In the sequel we will use the notation $\theta_{r}=\int_{B}\|x\|^{r} p_{1}(d x)$. It is a consequence of a theorem proved recently by Fernique that $\theta_{r}<\infty$ for $1 \leqq r<\infty$. Thus we have, for instance, $\mathscr{E}\left(\|W(t)-W(s)\|^{2}\right)=$ $|t-s| \theta_{2}$.

We will assume the following on $(i, H, B)$ : There exists a sequence $Q_{n}$ of finite dimensional projections such that $(1) Q_{n}(B) \subset B^{*}$, (2) $Q_{n}$ converges strongly to the identity both in $B$ and in $H$. It follows by the Principle of Uniform Boundedness that there exists a finite constant $\alpha>0$ such that $\left\|Q_{n}\right\|_{B, B}=\sup _{x \neq 0}\left\|Q_{n} x\right\| /\|x\|<\alpha$ for all $n$.

\section{Stochastic integrals.}

Notation. $\quad-\mathscr{l}_{t} \equiv \sigma$-field generated by $W(s), 0 \leqq s \leqq t . \quad \mathscr{B}(X, Y) \equiv$ the Banach space of all continuous linear operators from a Banach space $X$ into another Banach space $Y . \mathscr{B}_{1}(H, H)=$ the Banach space of all trace class operators of $H . \mathscr{\mathscr { B }}_{2}(H, H)=$ the Hilbert space of all Hilbert-Schmidt operators of $H$. Norms in $\mathscr{B}(X, Y), \mathscr{B}_{1}(H, H)$ and $\mathscr{B}_{2}(H, H)$ are denoted by $\|\cdot\|_{X, Y},\|\cdot\|_{1}$ and $\|\cdot\|_{2}$ respectively. $c$ will always denote a constant such that $\|x\| \leqq c|x|$ for all $x$ in $H$.

REMARK 3.1. Every bounded operator from $B$ into $B^{*}$ is a trace 
class operator of $H$ (p. 10 in [9]). In fact we have $\|S\|_{1} \leqq \theta_{2}\|S\|_{B, B^{*}}$ for all $S \in \mathscr{B}\left(B, B^{*}\right)$. A map from $B$ into itself with image in $B^{*}$ will be called skinny.

DEFINITION 3.1. By a non-anticipating skinny transformation (n.a.s.t.) we mean a stochastic process $\zeta(t, \omega)(t \in[0, \infty), \omega \in \Omega)$ with state space $\mathscr{B}\left(B, B^{*}\right)$ with the properties that $\zeta$ is $(t, \omega)$-jointly measurable and for each $t \geqq 0 \zeta(t, \cdot)$ is $\mathscr{C l}_{t}$-measurable. $\zeta$ is said to be simple if there is a partition $\left\{0=t_{0}<t_{1}<\cdots<t_{n}\right\}$ such that $\zeta(t, \omega)=$ $\zeta\left(t_{j}, \omega\right)$ for $t_{j} \leqq t<t_{j+1}, j=0,1, \cdots, n-1$, and $\zeta(t, \omega)=\zeta(t, \omega)$ for $t_{n} \leqq t$.

By using the same technique of Lemma 7.1 in [8] we can easily prove the following

Lemma 3.1. Let $\zeta$ be a n.a.s.t such that for each $0<T<\infty$

$$
\mathscr{E} \int_{0}^{T}\|\zeta(t, \omega)\|_{B, B^{*}}^{2} d t<\infty
$$

Then there exists a sequence $\zeta_{n}$ of simple n.a.s.t. such that for each $0<T<\infty$

$$
\lim _{n \rightarrow \infty} \mathscr{E} \int_{0}^{T}\left\|\zeta(t, \omega)-\zeta_{n}(t, \omega)\right\|_{B, B^{*}}^{2} d t=0
$$

Let $\zeta$ be a simple n.a.s.t. with jumps at $0<t_{1}<t_{2}<\cdots<t_{n}$. Suppose $\mathscr{E} \int_{0}^{T}\|\zeta(s, \omega)\|_{B, B^{*}}^{2} d s<\infty$ for each $0<T<\infty$. We define stochastic integral $I_{\zeta}$ of $\zeta$ with respect to $W(t)$ as follows:

$$
\begin{aligned}
I_{\zeta}(t, \omega)= & \sum_{k=0}^{j-1} \zeta\left(t_{k}, \omega\right)\left(W\left(t_{k+1}, \omega\right)-W\left(t_{k}, \omega\right)\right) \\
& +\zeta\left(t_{j}, \omega\right)\left(W(t, \omega)-W\left(t_{j}, \omega\right)\right)
\end{aligned}
$$

if $t_{j} \leqq t<t_{j+1}, j=0,1, \cdots, n$.

Here we use the convention that $t_{0}=0, t_{n+1}=\infty$.

Notation.

$$
I_{\zeta}(t, \omega)=\int_{0}^{t} \zeta(s, \omega) d W(s, \omega) .
$$

REMARK 3.2. We will consider $I_{\zeta}$ as a stochastic process with state space $H$.

Lemma 3.2. Suppose $\zeta$ is a n.a.s.t. Then we have

(i) for $s<t, \mathscr{E}\left(|\zeta(s)(W(t)-W(s))|^{2}\right)=(t-s) \mathscr{E}\left(\|\zeta(s)\|_{2}^{2}\right)$.

(ii) for $s<t<u<v, \mathscr{E}<\zeta(s)(W(t)-W(s)), \zeta(u)(W(v)-W(u))>=0$.

Proof. We prove (i) only since (ii) can be proved similarly. Let 
$\left\{Q_{n}\right\}$ be the projections given in the end of $\S 2$. Define a sequence of random variables $f_{m}$ on $\Omega$ by

$$
f_{m}=\left|Q_{m} \zeta(s)(W(t)-W(s))\right|^{2} .
$$

So

$$
\begin{aligned}
f_{m} & \leqq|\zeta(s)(W(t)-W(s))|^{2} \leqq c\|\zeta(s)(W(t)-W(s))\|_{B^{*}}^{2} \\
& \leqq c\|\zeta(s)\|_{B, B^{*}}^{2}\|W(t)-W(s)\|^{2} .
\end{aligned}
$$

Since $\zeta$ is non-anticipating, we have

$$
\begin{aligned}
\mathscr{E}\left(\|\zeta(s)\|_{B, B^{*}}^{2}\|W(t)-W(s)\|^{2}\right) & =\mathscr{E}\left(\|\zeta(s)\|_{B, B^{*}}^{2}\right) \cdot \mathscr{E}\left(\|W(t)-W(s)\|^{2}\right) \\
& =(t-s) \theta_{2} \mathscr{E}\left(\|\zeta(s)\|_{B, B^{*}}^{2}\right) .
\end{aligned}
$$

Thus $f_{m}$ is dominated by an integrable function. Obviously $f_{m}$ converges to $|\zeta(s)(W(t)-W(s))|^{2}$ a.e. By the Lebesgue dominated convergence theorem,

$$
\mathscr{E}\left(|\zeta(s)(W(t)-W(s))|^{2}=\lim _{m \rightarrow \infty} \mathscr{E}\left(\mid Q_{m} \zeta(s)\left(W(t)-\left.(W(s))\right|^{2}\right) .\right.\right.
$$

Now

$$
\begin{aligned}
\mathscr{E}\left(\left|Q_{m} \zeta(s)(W(t)-W(s))\right|^{2}\right) & =\mathscr{E}\left(\sum_{j=1}^{m}<\zeta(s)(W(t)-W(s)), e_{j}>^{2}\right) \\
& =\sum_{j=1}^{m} \mathscr{E}\left\{\left(W(t)-W(s), \zeta^{*}(s) e_{j}\right)^{2}\right\} .
\end{aligned}
$$

We will prove below that

$$
\mathscr{E}\left\{\left(W(t)-W(s), \zeta^{*}(s) e_{j}\right)^{2}\right\}=(t-s) \sum_{k=1}^{\infty} \mathscr{E}\left\{\left(\zeta^{*}(s) e_{j}, e_{k}\right)^{2}\right\}
$$

Then

$$
\begin{aligned}
\mathscr{E}\left(\left|Q_{m} \zeta(s)(W(t)-W(s))\right|^{2}\right) & =\sum_{j=1}^{m}(t-s) \sum_{k=1}^{\infty} \mathscr{E}\left\{\left(\zeta^{*}(s) e_{j}, e_{k}\right)^{2}\right\} \\
& =(t-s) \mathscr{E}\left(\sum_{j=1}^{m}\left|\zeta^{*}(s) e_{j}\right|^{2}\right)
\end{aligned}
$$

which yields the desired conclusion when $m \rightarrow \infty$.

Let us prove now the assertion (1). Define $g_{n}=\left(Q_{n}(W(t)-W(s))\right.$, $\left.\zeta^{*}(s) e_{j}\right)^{2}$. Then $g_{n}$ converges to $\left(W(t)-W(s), \zeta^{*}(s) e_{j}\right)^{2}$ a.e. Furthermore $g_{n}$ is dominated by an integrable function since:

$$
\begin{aligned}
g_{n} & \leqq\left\|Q_{n}\right\|_{B, B}^{2}\|W(t)-W(s)\|^{2}\left\|\zeta^{*}(s)\right\|_{B, B^{*}}^{2}\left\|e_{j}\right\| \\
& \leqq \alpha^{2}\|W(t)-W(s)\|^{2}\left\|\zeta^{*}(s)\right\|_{B, B^{*}}^{2}\left\|e_{j}\right\| .
\end{aligned}
$$

Therefore by Lebesgue's convergence theorem, 


$$
\left.\mathscr{E}\left\{\left(W(t)-W(s), \zeta^{*}(s) e_{j}\right)^{2}\right\}=\lim _{n \rightarrow \infty} \mathscr{E}\left\{Q_{n}(W(t)-W(s)), \zeta^{*}(s) e_{j}\right)^{2}\right\} .
$$

Assertion (1) follows immediately by noting that

$$
\begin{aligned}
\mathscr{E}\left\{\left(Q_{n}(W(t)-W(s)), \zeta^{*}(s) e_{j}\right)^{2}\right\} \\
\quad=\mathscr{E}\left\{\left[\sum_{k=1}^{n}\left(W(t)-W(s), e_{k}\right)\left(\zeta^{*}(s) e_{j}, e_{k}\right)\right]^{2}\right\} \\
\quad=(t-s) \sum_{k=1}^{n} \mathscr{E}\left\{\left(\zeta^{*}(s) e_{j}, e_{k}\right)^{2}\right\}
\end{aligned}
$$

by a simple calculation.

Proposition 3.1. The stochastic integral $I_{\zeta}$ has the following properties:

(1) $I_{\zeta}$ is coutinuous in $t$ for almost all $\omega$.

(2) $I_{\zeta}$ is a martingale with respect to $\left\{\mathscr{L}_{t}\right\}$.

(3) $\mathscr{P}\left\{\sup _{0 \leqq t \leqq T}\left|I_{\zeta}(t, \omega)\right|>\alpha\right\} \leqq\left(1 / \alpha^{2}\right) \mathscr{E}\left\{\left|I_{\zeta}(T, \omega)\right|^{2}\right\}$

(4) $\mathscr{E}\left\{I_{\zeta}(t, \omega)\right\}=0, \mathscr{E}\left\{\left|I_{\zeta}(t, \omega)\right|^{2}\right\}=\mathscr{E} \int_{0}^{t}\|\zeta(s, \omega)\|_{2}^{2} d s$

(5) $I_{\alpha \zeta_{1}+\beta \zeta_{2}}=\alpha I_{\zeta_{1}}+\beta I_{\zeta_{2}}$, where $\zeta_{1}$ and $\zeta_{2}$ are n.a.s.t. and $\alpha, \beta \in R$.

(6) $I_{\zeta}$ is non-anticipating.

Proof. (1), (2), (5) and (6) are obvious, while (3) follows from (1) and (2) by Doob's inequality. Therefore we need only show (4). Without loss of generality we may assume that $t=t_{j+1}$ for some $j$. Thus

$$
\begin{aligned}
I_{\zeta}(t, \omega)= & \sum_{k=0}^{j} \zeta\left(t_{k}, \omega\right)\left(W\left(t_{k+1}, \omega\right)-W\left(t_{k}, \omega\right)\right) \\
\mid I_{\zeta}\left(t,\left.\omega\right|^{2}\right)= & \sum_{k=0}^{j} \sum_{m=0}^{j}<\zeta\left(t_{k}, \omega\right)\left(W\left(t_{k+1}, \omega\right)\right) \\
& -W\left(t_{k}, \omega\right), \zeta\left(t_{m}, \omega\right)\left(W\left(t_{m+1}, \omega\right)-W\left(t_{m}, \omega\right)\right)>.
\end{aligned}
$$

Apply Lemma 3.2 and we obtain

$$
\begin{aligned}
\mathscr{E}\left(\left|I_{\zeta}(t, \omega)\right|^{2}\right) & =\sum_{k=0}^{j}\left(t_{k+1}-t_{k}\right) \mathscr{E}\left(\left\|\zeta\left(t_{k}, \omega\right)\right\|_{2}^{2}\right) \\
& =\mathscr{E} \int_{0}^{t}\|\zeta(s, \omega)\|_{2}^{2} d s .
\end{aligned}
$$

Finally using the same argument used in the proof of Lemma 3.2 we see immediately that $\mathscr{E}\left\langle I_{\zeta}(t, \omega), h\right\rangle=0$ for all $h \in H$. Hence

$$
\mathscr{E}\left\{I_{\zeta}(t, \omega)\right\}=0
$$

Using Lemma 3.1 and applying the same technique used in 
Theorem 8 of [8], we can easily show the following.

THEOREM 3.1. For every n.a.s.t. $\zeta$ such that $\mathscr{E} \int_{0}^{T}\|\zeta(t, \omega)\|_{B, B^{*}}^{2} d t<$ $\infty$ for each $0<T<\infty$, we can determine a stochastic process

$$
I_{\zeta}(t, \omega) \equiv \int_{0}^{t} \zeta(s, \omega) d W(s, \omega)
$$

such that the properties (1)-(6) of Proposition 3.1 hold.

DEFINITION 3.2. By a non-anticipating transformation (n.a.t.) we mean a stochastic process $\xi(t, \omega)(t \in[0, \infty), \omega \in \Omega)$ with state space $\mathscr{B}(B, B)$ such that $\zeta(t, \omega)=\xi(t, \omega)-I$ in a n.a.s.t.

DEFINITION 3.3. If $\xi(t, w)$ is a n.a.t. such that

$$
\mathscr{E} \int_{0}^{T}\|\xi(t, \omega)-I\|_{B, B^{*}}^{2} d t<\infty \text { for each } 0<T<\infty,
$$

then we define the stochastic integral $I_{\xi}$ of $\xi$ with respect to $W$ by $I_{\hat{\xi}}(t, \omega)=W(t, \omega)+I_{\zeta}(t, \omega)$, where $\zeta=\xi-I$.

\section{Proposition 3.2.}

$$
\mathscr{E}\left\{\left\|I_{\xi}(t, \omega)\right\|^{2}\right\} \leqq 2(1+c)^{2}\left(1+\theta_{2}\right) \mathscr{E} \int_{0}^{t}\left(1+\|\zeta(s, \omega)\|_{2}^{2}\right) d s .
$$

Proof. Direct computation.

Definition 3.4. By a non-anticipating vector (n.a.v.) we mean a stochastic process $\sigma(t, \omega) \quad(t \in[0, \infty), \omega \in \Omega)$ with state space $H$ such that $\sigma$ is $(t, \omega)$-jointly measurable and for each $t \geqq 0, \sigma(t, \cdot)$ is $\mathscr{C}_{t^{-}}$ measurable. $\sigma$ is said to be simple if there is a finite partition of $[0, \infty)$ such that $\sigma$ is a constant random vector (i.e., with values in $H$ ) on each interval of the partition.

LEMma 3.3. Suppose $\sigma$ is a n.a.v. such that for each $0<T<\infty$ $\mathscr{E} \int_{0}^{T}|\sigma(t, \omega)|^{2} d t<\infty$. Then there exists a sequence of simple n.a.v. $\sigma_{n}$ such that $\sigma_{n} \in B^{*}$ and for each $0<T<\infty, \mathscr{E} \int_{0}^{T}\left|\sigma(t, \omega)-\sigma_{n}(t, \omega)\right|^{2} d t \rightarrow$ 0 as $n \rightarrow \infty$.

Proof. The same argument in Lemma 7.1 [8] shows that there is a sequence $\eta_{n}$ of simple n.a.v. such that for each $0<T<\infty$, $\mathscr{E} \int_{0}^{T}\left|\eta_{n}(t, \omega)-\sigma(t, \omega)\right|^{2} d t \rightarrow 0$ as $n \rightarrow \infty$.

Let $\left\{e_{k}\right\}$ be an orthonormal basis of $H$ lying entirely in $B^{*}$. Let 
$P_{n}$ be the orthogonal projection onto the span of $e_{1}, \cdots, e_{n}$. It is easy to see that $\sigma_{n}=P_{n} \eta_{n}$ is a desired sequence.

Let $\sigma$ be a simple n.a.v. in $B^{*}$ with jumps at $0<t_{1}<\cdots<t_{n}$. The stochastic integral $J_{\sigma}$ of $\sigma$ with respect to $W$ is defined as follows.

$$
\begin{aligned}
J_{\sigma}(t, \omega)= & \sum_{k=0}^{j-1}\left(\sigma\left(t_{k}, \omega\right), W\left(t_{k+1}, \omega\right)-W\left(t_{k}, \omega\right)\right) \\
& +\left(\sigma\left(t_{j}, \omega\right), W(t, \omega)-W\left(t_{j}, \omega\right)\right), t_{j} \leqq t<t_{j+1}
\end{aligned}
$$

where $t_{0}=0, t_{n+1}=\infty$.

$$
\text { Notation. } \quad J_{\sigma}(t, \omega) \equiv \int_{0}^{t}(\sigma(s, \omega), d W(s, \omega)) .
$$

REMARK 3.3. Recall that (,) is the natural pairing of $B^{*}$ and $B$. Hence $J_{\sigma}$ is a stochastic process with values in $R$.

Parallel to $I_{\zeta}$ the stochastic integral $J_{\sigma}$ has the similar Properties and extensions. We will omit the proof since it is routine.

THEOREM 3.2. For every n.a.v. $\sigma$ such that $\mathscr{E} \int_{0}^{T}|\sigma(t, \omega)|^{2} d t<\infty$ for each $0<T<\infty$; we can determine a stochastic process $J_{\sigma}(t, \omega) \equiv$ $\int_{0}^{t}(\sigma(s, \omega), d W(s, \omega))$ such that

(1) $J_{\sigma}$ is continuous in $t$ for almost all $\omega$

(2) $J_{\sigma}$ is a martingale with respect to $\left\{\mathscr{L}_{t}\right\}$

(3) $\mathscr{P}\left\{\sup _{0 \leqq t \leqq T}\left|J_{\sigma}(t, \omega)\right|>\alpha\right\} \leqq\left(1 / \alpha^{2}\right) \mathscr{E}\left\{J_{\sigma}(T, \omega)^{2}\right\}$

(4) $\mathscr{E} J_{\sigma}(t, \omega)=0, \mathscr{E}\left\{J_{\sigma}(t, \omega)^{2}\right\}=\mathscr{E} \int_{0}^{t}|\sigma(s, \omega)|^{2} d s$

(5) $J_{\alpha \sigma_{1}+\beta \sigma_{2}}=\alpha J_{\sigma_{1}}+\beta J_{\sigma_{2}}, \quad \alpha, \beta \in R$

(6) $J_{o}$ is also non-anticipating.

REMARK 3.4. The reader should not be surprised that we can determine $J_{\sigma}$ such that it is continuous in $t$ for almost all $\omega$. Consider for example the simplest case $\sigma \equiv h \in H \backslash B^{*}$. The stochastic process $(h, W(t))=X(t)$ is not continuous in $t$. However it has a continuous version. This can be seen by observing that

$$
\mathscr{E}\left\{(X(t)-X(s))^{4}\right\}=3(t-s)^{2}|h|^{4} .
$$

4. Ito's formula. Let $f$ be a real-valued function defined in an open set $U$ of $B$. We will consider two kinds of differentiability for $f$. The Frechet derivative of $f$ at $x \in U$ is the element $a(x) \in B^{*}$ such that

$$
|f(x+y)-f(x)-(a(x), y)|=o(\|y\|) \quad \text { for small } y \in B .
$$


We will always denote $a(x)$ by $f^{\prime}(x) . \quad f$ is said to be of class $C^{1}$ if $f^{\prime}(x)$ exists for every $x \in U$ and $f^{\prime}$ is continuous from $U$ into $B^{*}$. On the other hand, $f$ is said to be Frechet differentiable at $x$ in $H$ directions (briefly, $H$-differentiable at $x$ ) if there exists an element $b(x) \in H$ such that

$$
|f(x+h)-f(x)-\langle b(x), h\rangle|=0(|h|) \quad \text { for small } h \in H .
$$

$b(x)$ is easily seen to be unique and will be denoted always by $D f(x)$. Note that the existence of $f^{\prime}(x)$ implies that of $D f(x)$ and $f^{\prime}(x)=$ $D f(x)$, but the existence of $D f(x)$ does not imply the continuity of $f$ at $x$ in $B$-topology. Inductively we can define $f^{(n)}$, class $C^{n}$ and $D^{n} f$.

Theorem 4.1. (Ito's Formula). Let $f(t, x)$ be a real-valued continuous function on $[0, \infty) \times B$. Suppose

(1) for each $x \in B, f(\cdot, x)$ is of class $C^{1}$ and $\partial f / \partial s$ is continuous on $[0, \infty) \times B$.

(2) for each $t \geqq 0, f(t, \cdot)$ is twice $H$-differentiable with $D^{2} f(t, x) \in$ $\mathscr{B}_{1}(H, H)$ for all $x \in B$.

(3) Df is continuous from $(0, \infty) \times B$ into $H$ and $D^{2} f$ is continuous from $(0, \infty) \times B$ into $\mathscr{B}_{1}(H, H)$. There exists $\delta>0$ such that $\int_{0}^{\delta}|D f(s, x)| d s<\infty, \int_{0}^{\delta}\left\|D^{2} f(s, x)\right\|_{1} d s<\infty$ for all $x$ in $B$. If $X(t, \omega)=x_{0}+\int_{0}^{t} \xi(s, \omega) d W(s, \omega)+\int_{0}^{t} \sigma(s, \omega) d s$, where $\xi$ is a n.a.t. and $\sigma$ is a n.a.v., then

$$
\begin{aligned}
f(t, X(t, \omega))= & f\left(0, x_{0}\right)+\int_{0}^{t}\left(\xi^{*}(s, \omega) D f(s, X(s, \omega)), d W(s, \omega)\right) \\
& +\int_{0}^{t}\left\{\frac{\partial f}{\partial s}(s, X(s, \omega))+<D f(s, X(s, \omega)), \sigma(s, \omega)>\right. \\
& \left.+\frac{1}{2} \operatorname{trace}\left[\xi^{*}(s, \omega) D^{2} f(s, X(s, \omega)) \xi(s, \omega)\right]\right\} d s
\end{aligned}
$$

where * denotes the adjoint of an operator when it is restricted to $H$.

THEOREM 4.2. Let $f(t, x)$ be a continuous function on $[0, \infty) \times$ $B, C^{1}$ in the $t$ variable and $C^{2}$ in the $x$ variable and satisfying the condition (3) of Theorem 4.1. Then the same formula as in Theorem 4.1 holds.

Proof of Theorem 4.1 by assuming Theorem 4.2:

Define $g_{n}(t, x)=f\left(t, Q_{n} x\right), n=1,2, \cdots$. It is easily checked that each $g_{n}$ satisfies the hypothesis of Theorem 4.2, $\partial g_{n}(t, x) / \partial t=\partial f\left(t, Q_{n} x\right) / \partial t$, $g_{n}^{\prime}(t, x)=Q_{n}^{*} D f\left(t, Q_{n} x\right)$ and $g_{n}^{\prime \prime}(t, x)=Q_{n}^{*} \circ D^{2} f\left(t, Q_{n} x\right) \circ Q_{n}$. Therefore by 
Theorem 4.2 we have

$$
\begin{aligned}
& f\left(t, Q_{n} X(t, \omega)\right) \\
&= f\left(0, Q_{n} x_{0}\right)+\int_{0}^{t}\left(\xi^{*}(s, \omega) Q_{n}^{*} D f\left(s, Q_{n} X(s, \omega)\right), d W(s, \omega)\right) \\
&+\int_{0}^{t}\left\{\frac { \partial f } { \partial s } \left(s, Q_{n} X(s, \omega)+<Q_{n}^{*} D f\left(s, Q_{n} X(s, \omega)\right), \sigma(s, \omega)>\right.\right. \\
&\left.+\frac{1}{2} \operatorname{trace}\left[\xi^{*}(s, \omega) Q_{n}^{*} \circ D^{2} f\left(s, Q_{n} X(s, \omega)\right) Q_{n} \xi(s, \omega)\right]\right\} d s .
\end{aligned}
$$

Letting $n \rightarrow \infty$, we get the desired conclusion.

The remainder of this section is devoted to the proof of Theorem 4.2. For the sake of notational convenience, we will prove Proposition 4.1 only. The proof of Theorem 4.2 follows similarly.

Lemma 4.1. Let $\zeta$ be a n.a.s.t. Then

(i) for $s \leqq t$,

$$
\begin{aligned}
\mathscr{E}\left\{(\zeta(s)(W(t)-W(s)), W(t)-W(s))^{2}\right\}= & (t-s)^{2} \mathscr{E}\left\{\|\zeta(s)\|_{2}^{2}\right. \\
& \left.+(\operatorname{trace} \zeta(s))^{2}\right\}
\end{aligned}
$$

(ii) for $s \leqq t \leqq u \leqq v$,

$$
\begin{aligned}
& \mathscr{E}\{(\zeta(s)(W(t)-W(s)), W(t)-W(s))\} \times \\
& \quad \times\{(\zeta(u)(W(v)-W(u)), W(v)-W(u))\} \\
& \quad=(v-u) \mathscr{E}\{\operatorname{trace} \zeta(u)(\zeta(s)(W(t)-W(s)), W(t)-W(s))\} .
\end{aligned}
$$

Proof. Direct computation.

Lemma 4.2. Let $\zeta$ be a n.a.s.t. Assume that $\zeta(t, \omega)$ is continuous in $t$ for almost all $\omega$ and $\|\zeta(t, \omega)\|_{B, B^{*}} \leqq M$ for all $t$ and a.e. $\omega$. $\pi_{n}=$ $\left\{0=t_{0}<t_{1}<\cdots<t_{n}=t\right\}$ is a partition of $[0, t] .\left|\pi_{n}\right|=\max _{0 \leqq j \leqq n-1}\left(t_{j+1}-t_{j}\right)$. Then

$$
\sum_{j=0}^{n-1}\left(\zeta\left(t_{j}, \omega\right)\left(W\left(t_{j+1}, \omega\right)-W\left(t_{j}, \omega\right)\right), W\left(t_{j+1}, \omega\right)-W\left(t_{j}, \omega\right)\right)
$$

converges in the mean to $\int_{0}^{t} \operatorname{trace} \zeta(s, \omega) d s$ as $\left|\pi_{n}\right| \rightarrow 0$.

Proof. For the sake of convenience we use the notation $\Delta t_{j}=$ $t_{j+1}-t_{j}, \Delta W_{j}=W\left(t_{j+1}, \omega\right)-W\left(t_{j}, \omega\right), S_{n}(t, \omega)=\sum_{\jmath=0}^{n-1}\left(\zeta\left(t_{j}, \omega\right) \Delta W_{j}, \Delta W_{j}\right)$ and $R_{n}(t, \omega)=\sum_{j=0}^{n-1} \Delta t_{j}$ trace $\zeta\left(t_{j}, \omega\right)$. Note first that since $\zeta$ is continuous we have $R_{n}(t, \omega) \rightarrow \int_{0}^{t} \operatorname{trace} \zeta(s, \omega) d s$ with $\mathscr{P}$-probability 1 as $\left|\pi_{n}\right| \rightarrow 0$. Using the assumption in $\zeta$ and the Lebesgue dominated 
convergence theorem it is easy to see that $R_{n}(t, \omega)$ converges to $\int_{0}^{t} \operatorname{trace} \zeta(s, \omega) d s$ in the mean as $\left|\pi_{n}\right| \rightarrow 0$. Therefore to finish the proof it is sufficient to show that $\mathscr{E}\left|S_{n}-R_{n}\right|^{2} \rightarrow 0$ as $\left|\pi_{n}\right| \rightarrow 0$.

$$
\begin{aligned}
\left|S_{n}-R_{n}\right|^{2}= & \left|\sum_{j=0}^{n-1}\left(\zeta\left(t_{j}, \omega\right) \Delta W_{j}, \Delta W_{j}\right)-\Delta t_{j} \operatorname{trace} \zeta\left(t_{j}, \omega\right)\right|^{2} \\
= & \sum_{j=0}^{n-1}\left\{\left(\zeta\left(t_{j}, \omega\right) \Delta W_{j}, \Delta W_{j}\right)-\Delta t_{j} \operatorname{trace} \zeta\left(t_{j}, \omega\right)\right\}^{2} \\
& +2 \sum_{i<j}\left\{\left(\zeta\left(t_{i}, \omega\right) \Delta W_{i}, \Delta W_{i}\right)-\Delta t_{i} \operatorname{trace} \zeta\left(t_{i}, \omega\right)\right\} \\
& \cdot\left\{\left(\zeta\left(t_{j}, \omega\right) \Delta W_{j}, \Delta W_{j}\right)-\Delta t_{j} \operatorname{trace} \zeta\left(t_{j}, \omega\right)\right\} .
\end{aligned}
$$

Applying Lemma 4.1, we obtain

$$
\begin{aligned}
\mathscr{E}\left|S_{n}-R_{n}\right|^{2} & =\sum_{j=0}^{n-1} \Delta t_{j}^{2} \mathscr{E}\left\{\left\|\zeta\left(t_{j}, \omega\right)\right\|_{2}^{2}\right\} \\
& \leqq \sum_{j=0}^{n-1} \Delta t_{j}^{2} \theta_{2} \mathscr{E}\left\{\left\|\zeta\left(t_{j}, \omega\right)\right\|_{B, B^{*}}^{2}\right\} \\
& \leqq \theta_{2} M^{2} \sum_{j=0}^{n-1} \Delta t_{j}^{2} \leqq \theta_{2} M^{2} t\left|\pi_{n}\right| \rightarrow 0 \text { as }\left|\pi_{n}\right| \rightarrow 0
\end{aligned}
$$

Proposition 4.1. If $f$ is a real-valued function of class $C^{2}$ on $B$, then

$$
\begin{aligned}
f(W(t, \omega))= & f(0)+\int_{0}^{t}\left(f^{\prime}(W(s, \omega)), d W(s, \omega)\right) \\
& +\frac{1}{2} \int_{0}^{t} \operatorname{trace} f^{\prime \prime}(W(s, \omega)) d s .
\end{aligned}
$$

Proof. Obviously the integrals have continuous versions. Therefore it suffices to prove the equallity for each fixed $t$. Because $f$ is $C^{2}$, we have

$$
\begin{aligned}
f(x)-f(y)= & \left(f^{\prime}(y), x-y\right)+\frac{1}{2}\left(f^{\prime \prime}(y)(x-y), x-y\right) \\
& +o\left(\|x-y\|^{2}\right) .
\end{aligned}
$$

Recall that (, ) is the natural pairing of $B^{*}$ and $B$. Note that $f^{\prime}(y) \in$ $B^{*}$ and $f^{\prime \prime}(y) \in \mathscr{B}\left(B, B^{*}\right)$ for all $y \in B$. We may assume that $\left\|f^{\prime \prime}(y)\right\|_{B, B^{*}} \leqq M$ for all $y \in B$. Let $\pi_{n}=\left\{0=t_{0}<t_{1}<\cdots<t_{n}=t\right\}$ be a partition of $[0, t]$ and $\left|\pi_{n}\right|=\max _{0 \leqq j \leqq n-1}\left(t_{j-1}-t_{j}\right)$.

Using (1) we obtain

$$
\begin{aligned}
f(W(t, \omega))-f(0) & =\sum_{j=0}^{n-1} f\left(W\left(t_{j+1}, \omega\right)\right)-f\left(W\left(t_{j}, \omega\right)\right) \\
& =\sum_{j=0}^{n-1}\left(f^{\prime}\left(W\left(t_{j}, \omega\right)\right), W\left(t_{j+1}, \omega\right)-W\left(t_{j}, \omega\right)\right)
\end{aligned}
$$




$$
\begin{aligned}
& +\sum_{j=0}^{n-1} \frac{1}{2}\left[f ^ { \prime \prime } ( W ( t _ { j } , \omega ) ) \left(W\left(t_{j+1}, \omega\right)\right.\right. \\
& \left.\left.-W\left(t_{j}, \omega\right)\right), W\left(t_{j+1}, \omega\right)-W\left(t_{j}, \omega\right)\right] \\
& +\sum_{j=0}^{n-1} o\left(\left\|W\left(t_{j+1}, \omega\right)-W\left(t_{j}, \omega\right)\right\|^{2}\right)
\end{aligned}
$$

$$
\begin{aligned}
\mathscr{E} \mid \sum_{j=0}^{n-1} & \left(f^{\prime}\left(W\left(t_{j}, \omega\right)\right), W\left(t_{j+1}, \omega\right)-W\left(t_{j}, \omega\right)\right) \\
& -\left.\int_{0}^{t}\left(f^{\prime}(W(s, \omega)), d W(s, \omega)\right)\right|^{2} \\
= & \mathscr{E}\left|\sum_{j=0}^{n-1} \int_{t_{j}}^{t_{j+1}}\left(f^{\prime}\left(W\left(t_{j}, \omega\right)\right)-f^{\prime}(W(s, \omega)), d W(s, \omega)\right)\right|^{2} \\
= & \mathscr{E} \sum_{j=0}^{n-1} \sum_{i=0}^{n-1} \int_{t_{j}}^{t_{j+1}} \int_{t_{i}}^{t_{i+1}} .
\end{aligned}
$$

Now use (4) of Proposition 3.1 to conclude that the above quantity is equal to

$$
\begin{aligned}
& \sum_{\jmath=0}^{n-1} \int_{t_{j}}^{t_{j+1}} \mathscr{E} \mid f^{\prime}\left(W\left(t_{j}, \omega\right)-\left.f^{\prime}(W(s, \omega))\right|^{2} d s\right. \\
& \leqq \sum_{j=0}^{n-1} \int_{t_{j}}^{t_{j+1}} \mathscr{E}\left\{\left|f^{\prime \prime}\left(W\left(t_{j}, \omega\right)\right)\left(W\left(t_{j}, \omega\right)-W(s, \omega)\right)\right|^{2}+o\left(\| W\left(t_{j}, \omega\right)\right.\right. \\
& \text { - W(s, } \omega)) \|)\} d s \\
& \leqq \sum_{j=0}^{n-1}\left[c^{2} M^{2} \theta_{2}\left(t_{j+1}-t_{j}\right)^{2}+o\left(\left|t_{j+1}-t_{j}\right|^{1 / 2}\right)\left(t_{j+1}-t_{j}\right)\right] \rightarrow 0 \text { as }\left|\pi_{n}\right| \rightarrow 0 \text {. }
\end{aligned}
$$

Therefore $\sum_{j=0}^{n=1}\left(f^{\prime}\left(W\left(t_{j}, \omega\right)\right), W\left(t_{j+1}, \omega\right)-W\left(t_{j}, \omega\right)\right)$ converges to $\int_{0}^{t}\left(f^{\prime}(W(s, \omega)), d W(s, \omega)\right)$ in the mean as $\left|\pi_{n}\right|$ tends to zero.

(ii) By Lemma 4.2,

$$
\sum_{j=0}^{n-1} \frac{1}{2}\left(f^{\prime \prime}\left(W\left(t_{j}, \omega\right)\right)\left(W\left(t_{j+1}, \omega\right)-W\left(t_{j}, \omega\right)\right), W\left(_{j+1}, \omega\right)-\left(W\left(t_{j}, \omega\right)\right)\right.
$$

converges to $1 / 2 \int_{0}^{t} \operatorname{trace} f^{\prime \prime}(W(s, \omega)) d s$ in the mean as $\left|\pi_{n}\right|$ tends to zero.

(iii) It is easy to see that

$$
\begin{aligned}
\mathscr{E} \mid \sum_{j=0}^{n-1}\left\{|| W\left(t_{j+1}, \omega\right)-W\left(t_{j}, \omega\right) \|^{2}-\left.\theta_{2}\left(t_{j+1}-t_{j}\right\}\right|^{2}\right. \\
\quad=\left(\theta_{4}-\theta_{2}^{2}\right) \sum_{j=0}^{n-1}\left(t_{j+1}-t_{j}\right)^{2} \leqq\left(\theta_{4}-\theta_{2}^{2}\right) t\left|\pi_{n}\right| \rightarrow 0 \text { as }\left|\pi_{n}\right| \rightarrow 0 .
\end{aligned}
$$

Therefore

$$
\sum_{j=0}^{n-1} o\left(\left\|W\left(t_{j+1}, \omega\right)-W\left(t_{j}, \omega\right)\right\|^{2}\right)
$$


converges to 0 in the mean as $\left|\pi_{n}\right| \rightarrow 0$.

Finally, choose a subsequence of partitions such that the convergence in (i ), (ii) and (iii) is in the sense of almost sure. Then the desired conclusion follows immediately from (2).

5. Stochastic integral equation. Let $A$ be a map from $\left[t_{0}, \infty\right) \times$ $B, t_{0} \geqq 0$, into $\mathscr{B}(B, B)$ such that $(A(t, x)-I)(B) \subset B^{*}$ and $\sigma$ a map from $\left[t_{0}, \infty\right) \times B$ into $H$. Consider the stochastic integral equation

$$
X(t, \omega)=\nu(\omega)+\int_{t_{0}}^{t} A(s, X(s, \omega)) d W(s, \omega)+\int_{t_{0}}^{t} \sigma(s, X(s, \omega)) d s
$$

where $\nu$ is $\mathscr{C l}_{t_{0}}$-measurable.

Our objective is to seek a solution. In order to make the integrals meaningful, this solution must be non-anticipating with respect to $\left\{\mathscr{C l}_{t}\right\}$.

THEOREM 5.1. Assume that $A$ and $\sigma$ satisfy the following conditions

(i) $A(t, x)-I$ is continuous in $t$ from $\left[t_{0}, \infty\right)$ into $\mathscr{B}\left(B, B^{*}\right)$ for each $x \in B . \quad \sigma(t, x)$ is continuous in $t$ from $\left[t_{0}, \infty\right)$ into $H$ for each $x \in B$.

(ii) There exists a constant $K$ such that for all $t \geqq t_{0}$ and $x, y \in B$,

$$
\begin{gathered}
\|A(t, x)-A(t, y)\|_{2} \leqq K\|x-y\| \\
|\sigma(t, x)-\sigma(t, y)| \leqq K\|x-y\| \\
\|A(t, x)-I\|_{2}^{2} \leqq K\left(1+\|x\|^{2}\right) \\
|\sigma(t, x)|^{2} \leqq K\left(1+\|x\|^{2}\right) \\
\mathscr{E}\left(\|\nu(\omega)\|^{2}\right)<\infty .
\end{gathered}
$$

Then there exists a unique non-anticipating continuous solution of (3).

REMARK. Obviously it is sufficient to consider the case $t_{0} \leqq t \leqq T<$ $\infty$.

We will assume this in the following proof.

Proof. Let $\mathfrak{A}$ be the Banach space of all non-anticipating stochastic processes $X(t, \omega)$ with state space $B$ satisfying $\sup _{t_{0} \leqq t \leqq T} \mathscr{E}\left(\|X(t, \omega)\|^{2}\right)<$ $\infty$ with norm

$$
\|X X \mid\|=\left\{\sup _{t_{0} \leqq t \leqq T} \mathscr{E}\left(\|X(t, \omega)\|^{2}\right)\right\}^{1 / 2} .
$$

Observe that $W \in \mathfrak{U}$ and $\|W\|=\sqrt{\theta_{2} T} . \quad$ Define a map $\Phi$ in $\mathfrak{Y}$ by: $\quad X \in$ $\mathfrak{U}$ 


$$
\Phi(X)(t, \omega)=\nu(\omega)+\int_{t_{0}}^{t} A(s, X(s, \omega)) d W(s, \omega)+\int_{t_{0}}^{t} \sigma(s, X(s, \omega)) d s .
$$

Note that $A(s, X(s, \omega))$ is a n.a.t. and $\sigma(s, X(s, \omega)$ is a n.a.v. Therefore the integrals make sense. Moreover $\Phi(X)$ is clearly nonanticipating and the integrals exists by the assumption (ii). Furthermore, by (1) of Proposition 3.1, $\Phi(X)$ is a continuous process.

$$
\begin{aligned}
\Phi(X)(t, \omega)= & \nu(\omega)+W(t, \omega)-W\left(t_{0}, \omega\right)+\int_{t_{0}}^{t}(A(s, X(s, \omega))-I) d W(s, \omega) \\
& +\int_{t_{0}}^{t} \sigma(s, X(s, \omega)) d s .
\end{aligned}
$$

Use the inequality $\left(\sum_{i=1}^{5} a_{i}\right)^{2} \leqq 5 \sum_{i=1}^{5} a_{i}^{2}$ and apply (4) of Proposition 3.1 , it is easy to check that

$$
|\|\Phi(X)\||^{2} \leqq 5\left[a+2 \sqrt{\theta_{2} T}+c^{2} K^{2}\left(T-t_{0}\right)\left(1+T-t_{0}\right)\left(1+\|X \mid\|^{2}\right)\right],
$$

where $a=\mathscr{E}\left(\|\nu(\omega)\|^{2}\right)$. Hence $\Phi(X) \in \mathfrak{X}$ and $\Phi$ maps $\mathfrak{X}$ into itself.

Now let $X, Y \in \mathfrak{A}$,

$$
\begin{aligned}
(\Phi(X)-\Phi(Y))(t, \omega)= & \int_{t_{0}}^{t}(A(s, X(s, \omega))-A(s, Y(s, \omega))) d W(s, \omega) \\
& +\int_{t_{0}}^{t}(\sigma(s, X(s, \omega))-\sigma(s, Y(s, \omega))) d s
\end{aligned}
$$

Hence

$$
\begin{aligned}
\mathscr{E} \| & (\Phi(X)-\Phi(Y))(t, \omega) \|^{2} \\
\leqq & 2 c^{2} \mathscr{E} \int_{t_{\mathrm{r}}}^{t}|A(s, X(s, \omega))-A(s, Y(s, \omega))|_{2}^{2} d s \\
& +2 c^{2}\left(t-t_{0}\right) \mathscr{E} \int_{t_{0}}^{t}|\sigma(s, X(s, \omega))-\sigma(s, Y(s, \omega))|^{2} d s \\
\leqq & 2 c^{2} K^{2}\left(1+T-t_{0}\right) \int_{t_{0}}^{t} \mathscr{E}\left(\|X(s, \omega)-Y(s, \omega)\|^{2}\right) d s .
\end{aligned}
$$

Let $\alpha=2 c^{2} K^{2}\left(1+T-t_{0}\right)$. Then

$$
\mathscr{E}\|(\Phi(X)-\Phi(Y))(t, \omega)\|^{2} \leqq \alpha \int_{t_{0}}^{t} \mathscr{E}\left(\|X(s, \omega)-Y(s, \omega)\|^{2} d s .\right.
$$

Hence \|\|$\Phi(X)-\Phi(Y)\left\|\left|\leqq\left[\alpha\left(T-t_{0}\right)\right]^{1 / 2}\|X-Y \mid\|\right.\right.$ and $\Phi$ is a Lip-1 map, a priori $\Phi$ is a continuous map.

Furthermore, using (4) to get for any $m>1 \mathscr{E} \|\left(\Phi^{m}(X)-\right.$ $\left.\Phi^{m}(Y)\right)(t, \omega)\left\|^{2} \leqq\left[\left(\alpha\left(t-t_{0}\right)\right]^{n} / m !\right) \mid\right\| X-Y\|\|^{2}$. Thus $\left\|\Phi^{m}(X)-\Phi^{m}(Y)\right\| \mid \leqq$ $\left(\sqrt{\left[\alpha\left(T-t_{0}\right]^{m}\right.} / m !\right)\||X-Y|\|$. Let $0<\delta<1$ be fixed. Let $N$ be such that $\left(\sqrt{\left[\alpha\left(T-t_{0}\right)\right]^{m}} / m !\right)<\delta$ for all $m \geqq N$. Thus for all $m \geqq N$, $\left\||| \Phi^{m}(X)-\Phi^{m}(Y)\right\|\left|<\delta\||| X-Y\|\right.$. That is, $\Phi^{m}$ is a contraction from $\mathfrak{U}$ into itself whenever $m \geqq N$. By the generalized contraction 
mapping theorem, $\Phi$ has a fixed point which solves the equation (3) by the definition of $\Phi$. This solution is a continuous process because $\Phi(X)$ is continuous for every $X \in \mathfrak{X}$. Finally we show (3) has a unique solution. Suppose $X$ and $Y$ are solutions of (3). Then $\Phi(X)=X$, $\Phi(Y)=Y$. Using the same argument in the derivation of the solution, it is easy to see that $X=Y$ in $\mathfrak{X}$. Therefore $X(t, \omega)=Y(t, \omega)$ a.e. for each $t$. But $X$ and $Y$ are continuous, so $X(t, \omega)=Y(t, \omega)$ for all $t$ with $\mathscr{P}$-probability 1 .

THEOREM 5.2. Suppose $A$ and $\sigma$ satisfy the conditions of Theorem 5.1. Then the process $X(t)$ which solves the stochastic integral equation $X(t)=X(0)+\int_{0}^{t} A(s, X(s, \omega)) d W(s, \omega)+\int_{0}^{t} \sigma(s, X(s, \omega)) d s$ is a Markov process with transition probability $q(t, x, s, E)=\mathscr{P}\{X(s) \in E \mid X(t)=$ $x\}$. Moreover, $X(t)$ is homogeneous and satisfies the strong Markov property if $A$ and $\sigma$ are independent of $t$.

Proof. The first part can be shown in the same manner as [6] and [8]. We show the second part. Let $\psi_{t, x}(s, \omega), s \geqq t$, denote the solution of the stochastic integral equation,

$$
Y(s, \omega)=x+\int_{t}^{s} A(Y(u, \omega)) d W(u)+\int_{t}^{s} \sigma(Y(u, \omega)) d u .
$$

Let $\tau$ be any stopping time. Then

$$
X(s+\tau, \omega)=X(\tau, \omega)+\int_{\tau}^{s+\tau} A(X(u, \omega)) d W(u)+\int_{\tau}^{s+\tau} \sigma(X(u, \omega)) d u
$$

or

$$
X(s+\tau, \omega)=X(\tau, \omega)+\int_{0}^{s} A(X(v+\tau, \omega)) d W(v+\tau)+\int_{0}^{s} \sigma(\dot{X}(v+\tau, \omega)) d v .
$$

But $W(v+\tau)-W(\tau)$ is also a Wiener process in $B$ independent of $\mathscr{M}_{\tau}$. Now $\psi_{0, X(\tau)}(s, \omega)$ and $X(s+\tau, \omega)$ are both solutions of the last equation. By the uniqueness of solution, $\psi_{0, X(\tau)}(s, \omega)=X(s+\tau, \omega)$. Now let $E$ be a Borel set in $B$ and $x \in B$, then

$$
\begin{aligned}
\mathscr{E}^{x}\left(X(s+\tau) \in E \mid \mathscr{M}_{\tau}\right) & =\mathscr{E}^{x}\left(\psi_{0, X(\tau)}(s) \in E \mid \mathscr{M}_{\tau}\right) \\
& =\mathscr{E}(Y(s) \in E \mid Y(0)=X(\tau)) \\
& =q(s, X(\tau), E) .
\end{aligned}
$$

Hence $X$ is homogeneous and satisfies the strong Markov property.

We wish to thank Professor L. Gross and Professor K. Ito for their conversations and encouragement. 


\section{REFERENCES}

1. E. M. Cabana, On stochastic differentials in Hilbert spaces, Proc. Amer. Math. Soc., 20 (1969), 259-265.

2. Yu. L. Daletzky, Differential equations with functional derivatives and stochastic equations for generalized random processes (English translation), Soviet Math. Dokl., 7 (1966), 220-223.

3. L. Gross, Abstract Wiener space, Proc. of 5th Berkeley Symposium on Math. Stat. \& Prob. 2 (1965), 31-42.

4. - Potential theory on Hilbert space, J. Func. Anal., 1 (1967), 123-181.

5. K. Ito, Stochastic integral, Proc. Imp. Acad. Tokyo, 20 (1944), 519-524.

6. - Stochastic differential equations in a differentiable manifold, Nagoya Math. J., 1 (1950), 35-47.

7. — On a formula concerning stochastic differentials, Nagoya Math. J., 3 (1951), 55-65.

8. - On stochastic differential equations, Mem. Amer. Math. Soc., No. 4 (1951).

9. H. H. Kuo, Integration theory on infinite dimensional manifolds, Thesis, Cornell University (1970).

10. H. P. McKean, Stochastic integrals, Academic Press (1969).

Received November 23, 1970. Research supported by the National Science Foundation, Grant NSF-GP-19617

Courant Institute, New York University 



\section{PACIFIC JOURNAL OF MATHEMATICS}

\section{EDITORS}

\author{
H. SAMELSON \\ Stanford University \\ Stanford, California 94305

\section{R. HoвBY} \\ University of Washington \\ Seattle, Washington 98105
}

\section{J. DugundJI}

Department of Mathematics University of Southern California Los Angeles, California 90007

\author{
RichaRd ARENS \\ University of California \\ Los Angeles, California 90024
}

\section{ASSOCIATE EDITORS}
E. F. BECKENBACH
B. H. NEUMANN
F. WOLF
K. YoshidA

\section{SUPPORTING INSTITUTIONS}

\author{
UNIVERSITY OF BRITISH COLUMBIA \\ CALIFORNIA INSTITUTE OF TECHNOLOGY \\ UNIVERSITY OF CALIFORNIA \\ MONTANA STATE UNIVERSITY \\ UNIVERSITY OF NEVADA \\ NEW MEXICO STATE UNIVERSITY \\ OREGON STATE UNIVERSITY \\ UNIVERSITY OF OREGON \\ OSAKA UNIVERSITY
}

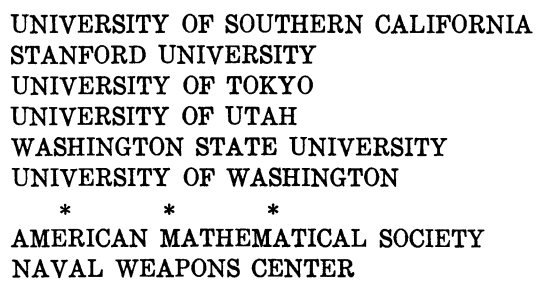

The Supporting Institutions listed above contribute to the cost of publication of this Journal, but they are not owners or publishers and have no responsibility for its content or policies.

Mathematical papers intended for publication in the Pacific Journal of Mathematics should be in typed form or offset-reproduced, (not dittoed), double spaced with large margins. Underline Greek letters in red, German in green, and script in blue. The first paragraph or two must be capable of being used separately as a synopsis of the entire paper. The editorial "we" must not be used in the synopsis, and items of the bibliography should not be cited there unless absolutely necessary, in which case they must be identified by author and Journal, rather than by item number. Manuscripts, in dup icate if possible, may be sent to any one of the four editors. Please classify according to the scheme of Math. Rev. Index to Vol. 39. All other communications to the editors should be addressed to the managing editor, Richard Arens, University of California, Los Angeles, California, 90024.

50 reprints are provided free for each article; additional copies may be obtained at cost in multiples of 50 .

The Pacific Journal of Mathematics is published monthly. Effective with Volume 16 the price per volume (3 numbers) is $\$ 8.00$; single issues, $\$ 3.00$. Special price for current issues to individual faculty members of supporting institutions and to individual members of the American Mathematical Society: $\$ 4.00$ per volume; single issues $\$ 1.50$. Back numbers are available.

Subscriptions, orders for back numbers, and changes of address should be sent to Pacific Journal of Mathematics, 103 Highland Boulevard, Berkeley, California, 94708.

PUBLISHED BY PACIFIC JOURNAL OF MATHEMATICS, A NON-PROFIT CORPORATION

Printed at Kokusai Bunken Insatsusha (International Academic Printing Co., Ltd.), 270, 3-chome Totsuka-cho, Shinjuku-ku, Tokyo 160, Japan. 


\section{Pacific Journal of Mathematics}

\section{Vol. 41, No. 2 December, 1972}

Tom M. (Mike) Apostol, Arithmetical properties of generalized Ramanujan sums .......................................... 281

David Lee Armacost and William Louis Armacost, On p-thetic groups ........ 295

Janet E. Mills, Regular semigroups which are extensions of groups .......... 303

Gregory Frank Bachelis, Homomorphisms of Banach algebras with minimal ideals ................................................ 307

John Allen Beachy, A generalization of injectivity .................. 313

David Geoffrey Cantor, On arithmetic properties of the Taylor series of rational functions. II.........................................

Václáv Chvátal and Frank Harary, Generalized Ramsey theory for graphs. III.

Small off-diagonal numbers .................................. 335

Frank Rimi DeMeyer, Irreducible characters and solvability of finite groups . . . . 347

Robert P. Dickinson, On right zero unions of commutative semigroups........ 355

John Dustin Donald, Non-openness and non-equidimensionality in algebraic

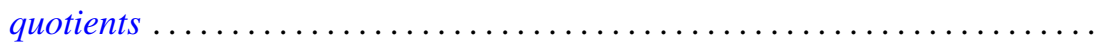

John D. Donaldson and Qazi Ibadur Rahman, Inequalities for polynomials with a prescribed zero ........................................ 375

Robert E. Hall, The translational hull of an $N$-semigroup ................ 379

John P. Holmes, Differentiable power-associative groupoids.............. 391

Steven Kenyon Ingram, Continuous dependence on parameters and boundary data for nonlinear two-point boundary value problems .

Robert Clarke James, Super-reflexive spaces with bases ..........

Gary Douglas Jones, The embedding of homeomorphisms of the plane in

continuous flows...............................

Mary Joel Jordan, Period $H$-semigroups and $t$-semisimple periodic

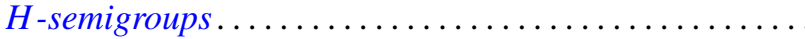

Ronald Allen Knight, Dynamical systems of characteristic 0

Kwangil Koh, On a representation of a strongly harmonic ring by sheaves...

Hui-Hsiung Kuo, Stochastic integrals in abstract Wiener space. ..

Thomas Graham McLaughlin, Supersimple sets and the problem of extending a

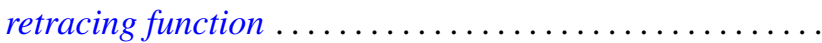

William Nathan, Open mappings on 2-manifolds .

M. J. O'Malley, Isomorphic power series rings

Sean B. O'Reilly, Completely adequate neighborhood systems and metrization

Qazi Ibadur Rahman, On the zeros of a polynomial and its derivative...

Russell Daniel Rupp, Jr., The Weierstrass excess function ..

Hugo Teufel, A note on second order differential inequalities and functional

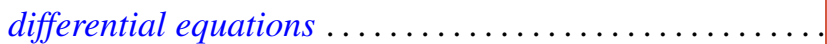

M. J. Wicks, A general solution of binary homogeneous equations over free 